\title{
Burden of Treatment-Induced Peripheral Neuropathy in Patients with Multiple Myeloma in Sweden
}

\author{
Hareth Nahi ${ }^{a, b}$ Göran Walinder ${ }^{a, b} \quad$ Vishal Patel $^{c}$ Ying Qu $^{d}$ Aaron Levine $^{d}$ \\ Istvan Majer ${ }^{c}$ Lucie Kutikovac $^{c}$ Eva Hellqvist Franck ${ }^{\mathrm{e}}$ Maria K. Svensson $^{\mathrm{e}} \mathrm{f}$ \\ Markus Hansson ${ }^{\mathrm{g}} \mathrm{h}$ \\ aHaematology Centre Karolinska, Karolinska University Hospital, Stockholm, Sweden; 'bepartment of Medicine, \\ Karolinska Institutet, Stockholm, Sweden; 'Amgen (Europe) GmbH, Rotkreuz, Switzerland; dIQVIA, Stockholm,

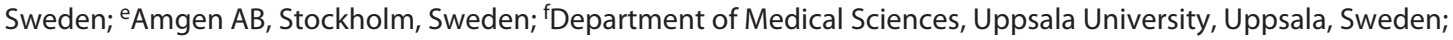

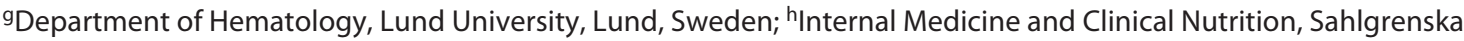 \\ Academy, Göteborg, Sweden
}

\section{Keywords}

Healthcare costs · Healthcare resource utilization · Multiple myeloma $\cdot$ Treatment-induced peripheral neuropathy

\begin{abstract}
Introduction: Treatment-induced peripheral neuropathy (TIPN) is a complication of multiple myeloma (MM) treatment. Objective: This real-world, retrospective study used electronic medical record (EMR) data from 3 Swedish clinics to assess the occurrence and economic burden of TIPN in patients with MM. Methods: Eligible patients had an MM diagnosis in the Swedish Cancer Registry between 2006 and 2015 and initiated treatment during that period. Follow-up was until last EMR visit, death, or study end (April 2017). The current analyses included patients receiving bortezomib, lenalidomide, carfilzomib, or thalidomide at any treatment line. To discern healthcare resource utilization (HCRU) and costs associated with TIPN from other causes, patients with TIPN were matched with those without on baseline characteristics, treatment, and line of therapy. All analyses were descriptive. Results: Overall, 457 patients were included; 102
\end{abstract}

karger@karger.com www.karger.com/aha

Karger $\stackrel{\text { ! }}{\div}$

GOPEN ACCESS
(C) 2021 The Author(s)

Published by S. Karger AG, Basel

This is an Open Access article licensed under the Creative Commons Attribution-NonCommercial-4.0 International License (CC BY-NC) (http://www.karger.com/Services/OpenAccessLicense), applicable to the online version of the article only. Usage and distribution for commercial purposes requires written permission.
(22\%) experienced TIPN. Patients experiencing TIPN during first-line treatment mostly received bortezomib-based regimens ( $n=48 / 57$ [84\%]); those with TIPN during second- and third/fourth-line treatment mostly received lenalidomide/ thalidomide-based regimens (19/31 [61\%], 8/14 [57\%], respectively). Patients with TIPN had higher HCRU/costs than those without TIPN (mean differences in hospital outpatient visits: $5.2, p=0.0031$; total costs per patient-year: EUR 17,183, $p=0.0007)$. Conclusions: Effective MM treatments associated with a reduced incidence of TIPN could result in decreased healthcare expenditure. $\odot 2021$ The Author(s)

Published by S. Karger AG, Basel

\section{Introduction}

Multiple myeloma (MM) accounts for approximately $1 \%$ of all cancers and is the second most common hematological malignancy after lymphoma [1-3]. The Association of the Nordic Cancer Registries reported that between 2012 and 2016, the age-standardized incidence of MM in Sweden was 3.7 per 100,000 for men and 2.4 per 
100,000 for women [3]. Five-year survival rates were 54 and $53 \%$, and $\mathrm{MM}$ accounted for 2.2 and $2.1 \%$ of all cancer deaths in men and women, respectively.

In Sweden, patients with MM are usually diagnosed and managed by physicians at hematology centers based in public hospitals, with treatment over recent years being guided by both national guidelines (http://www.sfhem. se/riktlinjer) and guidelines released by other organizations/working groups [4-6]. Since 2010, bortezomib and thalidomide have become part of standard induction regimens for patients aged $\leq 65$ years and for those aged $\geq 66$ years with good performance status, ahead of autologous stem cell transplantation (SCT) [7]. For those not eligible for SCT, melphalan, prednisone plus thalidomide, melphalan, prednisone plus bortezomib, lenalidomide plus dexamethasone, or bortezomib, lenalidomide plus dexamethasone are standard first-line treatment. However, treatments are often tailored for the individual patient, depending on characteristics such as patient age, comorbidity profile, and treatment history (in later lines). This is because in subsequent treatment lines it is considered best clinical practice to switch to a treatment with a different mechanism of action. Subsequent lines of therapy may also exacerbate pre-existing comorbidities and treatment toxicities.

Peripheral neuropathy is a common neurological complication associated with MM [8]. It causes debilitating symptoms including tingling, numbness, and neuropathic pain $[9,10]$, and can adversely impact patient quality of life $[11,12]$, functioning and/or performance $[13,14]$, and ability to work [15]. Peripheral neuropathy can be caused by $\mathrm{MM}$ itself and also by some of the agents used in the treatment of this disease [9]. In MM, treatment-induced peripheral neuropathy (TIPN) is particularly associated with bortezomib- and thalidomide-based regimens [16]. Intravenous bortezomib-based regimens are associated with TIPN in $34-54 \%$ of patients, with $8-16 \%$ experiencing grade 3 or higher events $[13,17,18]$. Although subcutaneously administered bortezomib is better tolerated than the intravenously administered drug [19-22], it is still associated with TIPN in $38 \%$ of patients and grade 3 or higher TIPN reported in $6 \%$ of patients [19]. The occurrence of TIPN can result in dose reductions and even therapy discontinuation $[1,11,23,24]$, which could potentially adversely impact patient outcomes. TIPN has also been shown to increase healthcare costs in patients with MM and other cancers $[25,26]$. Although TIPN is not as prevalent as it used to be, partly due to increased use of subcutaneous rather than intravenous bortezomib regimens, it remains an issue for some patients.
The incidence of TIPN in patients receiving MM treatment in clinical trials has been well documented, but the frequency of TIPN and the associated economic impact in clinical practice is less clear. This retrospective study aimed to assess the occurrence and economic burden of TIPN associated with the treatment of MM in a realworld setting in Sweden.

\section{Methods}

Study Design, Patients, and Objectives

This retrospective cohort study used electronic medical record (EMR) data from 3 large hematology clinics in Sweden (Karolinska University Hospital [Solna and Huddinge sites] and Stockholm South General Hospital [Södersjukhuset]), which were linked to national health registries (Swedish Cancer Registry, National Patient Register, National Prescribed Drug Register, and Cause of Death Register). Adults with a confirmed diagnosis of MM (according to ICD-10:CD90.0) in the Swedish Cancer Registry between 2006 and 2015 and who had initiated treatment during the same period were eligible for inclusion. Patients with missing treatment records for the study period and/or unclear treatment start date(s) were excluded. Patients were followed from the date of diagnosis until last EMR visit, death, or end of study (April 18, 2017), whichever occurred first.

Information on MM lines of therapy was retrieved from EMR case notes and free text using chemotherapeutic agents of interest as the search terms. The resultant data were categorized manually. If treatment start and/or finish dates were missing, these were determined based on visit dates, number of cycles of treatment received, or date of initiation of a new treatment. Where not explicitly stated, line of therapy was determined based on the gap between treatment administration dates (a gap of $>60$ days was considered a new line, while a gap of 60 days or less was considered a continuation of the same line). Maintenance treatment was considered a continuation of first-line treatment. Cases of peripheral neuropathy or TIPN were retrieved both from the National Patient Register via the relevant ICD-10 code (ICD-10:G62.0) and from a free-text search of the EMR case notes. Patients who had received bortezomib, lenalidomide, carfilzomib, or thalidomide at any line of treatment were eligible for inclusion in these analyses. The occurrence of selected pre-specified comorbidities (myocardial infarction, rheumatic diseases, congestive heart failure, diabetes with or without chronic complications, mild liver disease, renal disease, and monoclonal gammopathy of undetermined significance) was described for the period of 6 months prior to index date (defined as the date of first MM diagnosis).

The objectives of these analyses were to assess the occurrence of TIPN by treatment line and type of regimen, and the healthcare resource utilization (HCRU) and costs in patients with and without TIPN. A post hoc sub-analysis was also performed to assess whether there was a difference in the number of patients experiencing TIPN before and after the date when subcutaneous bortezomib became available for use in Sweden (September 28, 2012). The study was conducted in accordance with the Helsinki Declaration and was approved by an ethics committee (DNR 13280/20167). 
Table 1. Baseline patient characteristics stratified by TIPN status

\begin{tabular}{|c|c|c|c|}
\hline Variable & $\begin{array}{l}\text { All patients with } \\
\text { TIPN }(N=102)\end{array}$ & $\begin{array}{l}\text { All patients with no } \\
\text { TIPN }(N=355)\end{array}$ & $p$ value \\
\hline Sex, male, $n(\%)$ & $71(69.6)$ & $212(59.7)$ & 0.0698 \\
\hline Age, median (range), years & $66.0(40.0-86.0)$ & $68.0(23.0-93.0)$ & 0.0169 \\
\hline \multicolumn{4}{|l|}{ ISS stage, $n(\%)$} \\
\hline I & $12(11.8)$ & $51(14.4)$ & \multirow[t]{4}{*}{0.3920} \\
\hline II & $26(25.5)$ & $84(23.7)$ & \\
\hline III & $9(8.8)$ & $51(14.4)$ & \\
\hline Missing & $55(53.9)$ & $169(47.6)$ & \\
\hline \multicolumn{4}{|l|}{ Lab values, $[n]$ mean $(\mathrm{SD})$} \\
\hline Calcium, mg/dL & [58] $9.7(1.3)$ & [232] $9.5(1.1)$ & 0.7580 \\
\hline $\mathrm{Cr}, \mu \mathrm{mol} / \mathrm{L}$ & [62] $148.0(163.7)$ & [237] $149.1(198.9)$ & 0.9159 \\
\hline Hemoglobin, g/dL & [60] $11.8(1.8)$ & [246] $11.4(1.4)$ & 0.1569 \\
\hline B2-microglobulin, mg/L & {$[47] 4.9(5.0)$} & [186] $5.3(6.2)$ & 0.3435 \\
\hline Albumin, $\mathrm{g} / \mathrm{L}$ & [60] $32.2(5.4)$ & [235] $32.0(5.8)$ & 0.8979 \\
\hline Received SCT, $n(\%)$ & $45(44.1)$ & $126(35.5)$ & 0.1126 \\
\hline Prior history of TIPN, ${ }^{\mathrm{b}} n(\%)$ & $6(5.9)$ & $19(5.4)$ & 0.8356 \\
\hline
\end{tabular}

ISS, International Staging System; SCT, stem cell transplantation; SD, standard deviation; TIPN, treatmentinduced peripheral neuropathy. ${ }^{a}$ The Mann-Whitney U test or Kruskal-Wallis test was used for continuous variables and the $\chi^{2}$ test was used for categorical variables. ${ }^{\mathrm{b}}$ Patients with any evidence of prior TIPN in the available patient records.

Analyses

TIPN rates were assessed by type and line of MM treatment. HCRU was measured from TIPN diagnosis until the end of followup. To discern HCRU and costs associated with TIPN, from those resulting from other causes, a cohort of patients without TIPN was created. Patients with TIPN were matched with patients without TIPN on baseline characteristics (year of MM diagnosis, age group [in increments of 5 years], and sex), MM treatment that induced TIPN, and line of therapy. For patients with TIPN, the time from treatment initiation to TIPN diagnosis was estimated.

All cost calculations were based on 2015 Diagnosis Related Group (DRG) tariffs obtained from NordDRG (http://www.nordcase.org/eng/about-us/) and were converted from SEK to euro using 0.093535 as the exchange rate. Statistical Analysis System version 9.4 was used for data management and statistical analyses; all statistical analyses were descriptive in nature. The Mann-Whitney U test or Kruskal-Wallis test for continuous variables (mean and standard deviation) and the $\chi^{2}$ test for categorical variables were used to compare descriptive statistics between subgroups. $p$ values were not adjusted for multiplicity and so are included for hypothesis-generating purposes only.

\section{Results}

\section{Patients}

In total, 457 patients were eligible for inclusion in these analyses. Overall, 102 (22\%) patients experienced TIPN (Table 1$)$. Of these patients, $88(86 \%)$ had a minimum of 2 years history in their medical records, before initiation of the analyzed treatment line, and there were 6 cases of TIPN recorded in this 2 -year period. The remaining 14 patients did not have 2 years of prior history in their records and there were no cases of TIPN reported in the available history prior to initiating the analyzed line of treatment. Therefore, overall, 96 patients (94\%) had no prior evidence of TIPN in their medical records; the proportions of patients with prior history of TIPN were similar for those with (5.9\%) and without (5.4\%) TIPN in the present analysis. Patients with TIPN were younger than those without TIPN (median age 66 and 68 years, respectively; $p=0.0169$ ), and 70 and $60 \%$ of patients with and without TIPN, respectively, were men $(p=0.0698)$. International Staging System (ISS) stage was missing for 54 and $48 \%$ of those with and without TIPN, respectively. Of the remainder, ISS stage II was most commonly reported, being noted in 25 and $24 \%$ of patients with and without TIPN, respectively. Laboratory measurements such as calcium, Cr, hemoglobin, B2-microglobulin, and albumin levels were available for $46-61 \%$ of patients with and $52-69 \%$ of patients without TIPN. Where data were available, laboratory measurements appeared similar between these 2 groups of patients. Overall, 45/102 (44\%) patients with TIPN and 126/355 (35\%) patients without TIPN had received SCT. No patients had any of the pre-specified comorbidities during the 6 months prior to index date. 
Table 2. Treatment regimen and time to treatment initiation, stratified by line of treatment and TIPN status

\begin{tabular}{|c|c|c|c|c|c|c|}
\hline \multirow[t]{3}{*}{ Variable } & \multicolumn{6}{|c|}{ Treatment line in which patients received the regimen of interest, $n(\%)$} \\
\hline & \multicolumn{3}{|c|}{ All patients with TIPN $(N=102)$} & \multicolumn{3}{|c|}{ All patients with no TIPN $(N=355)$} \\
\hline & $\begin{array}{l}\text { first line } \\
(n=102)\end{array}$ & $\begin{array}{l}\text { second line } \\
(n=49)\end{array}$ & $\begin{array}{l}\text { third/fourth } \\
\text { line }(n=21)\end{array}$ & $\begin{array}{l}\text { first line } \\
(n=355)\end{array}$ & $\begin{array}{l}\text { second line } \\
(n=163)\end{array}$ & $\begin{array}{l}\text { third/fourth } \\
\text { line }(n=66)\end{array}$ \\
\hline \multicolumn{7}{|l|}{ Regimen, $n(\%)^{\mathrm{a}, \mathrm{b}}$} \\
\hline Bortezomib based & $68(66.7)$ & $18(36.7)$ & $8(38.1)$ & $246(69.3)$ & $47(28.8)$ & $27(40.9)$ \\
\hline Bortezomib + pomalidomide & $0(0.0)$ & $0(0.0)$ & $0(0.0)$ & $0(0.0)$ & $1(0.6)$ & $0(0.0)$ \\
\hline Bortezomib + thalidomide & $3(2.9)$ & $1(2.0)$ & $0(0.0)$ & $9(2.5)$ & $8(4.9)$ & $1(1.5)$ \\
\hline Carfilzomib based & $0(0.0)$ & $0(0.0)$ & $1(4.8)$ & $2(0.6)$ & $2(1.2)$ & $0(0.0)$ \\
\hline IMiD based & $20(19.6)$ & $29(59.2)$ & $10(47.6)$ & $103(29.0)$ & $92(56.4)$ & $29(43.9)$ \\
\hline Lenalidomide based & $5(4.9)$ & $25(51.0)$ & $10(47.6)$ & $44(12.4)$ & $79(48.5)$ & $20(30.3)$ \\
\hline Thalidomide based & $15(14.7)$ & $4(8.2)$ & $0(0.0)$ & $59(16.6)$ & $13(8.0)$ & $9(13.6)$ \\
\hline
\end{tabular}

IMiD, immunomodulatory drugs; MM, multiple myeloma; TIPN, treatment-induced neuropathy. ${ }^{\text {a }}$ Treatment groups are mutually exclusive. ${ }^{\mathrm{b}}$ Other chemotherapy and corticosteroids such as dexamethasone and prednisone may have been administered in combination with these regimens.

Table 3. Occurrence and time to TIPN, stratified by line of treatment and type of regimen inducing PN

\begin{tabular}{llcr}
\hline Type of regimen inducing PN & \multicolumn{2}{l}{ All patients with TIPN $(N=102)$} \\
\cline { 2 - 4 } & $\begin{array}{l}\text { TIPN during first } \\
\text { line }(n=57)\end{array}$ & $\begin{array}{l}\text { TIPN during second } \\
\text { line }(n=31)\end{array}$ & $\begin{array}{c}\text { TIPN during third/fourth } \\
\text { line }(n=14)\end{array}$ \\
\hline Bortezomib based, $n(\%)^{\mathrm{a}}$ & $48(84.2)$ & $12(38.7)$ & $6(42.9)$ \\
Time to TIPN, ${ }^{\mathrm{b}}$ median (range), months & $4.2(0.1-47.5)$ & $1.4(0.0-19.9)$ & $0.6(0.0-14.8)$ \\
IMiD based, $n(\%)^{\mathrm{c}}$ & $9(15.8)$ & $19(61.3)$ & $8(57.1)$ \\
Time to TIPN, ${ }^{\mathrm{b}}$ median (range), months & $2.0(0.0-17.0)$ & $1.1(0.0-38.0)$ & $4.8(0.0-36.9)$ \\
\hline
\end{tabular}

IMiD, immunomodulatory drugs; TIPN, treatment-induced peripheral neuropathy. ${ }^{\text {a }}$ Includes bortezomib-based and bortezomib plus lenalidomide/pomalidomide/thalidomide-based regimens. ${ }^{b}$ Time to first occurrence of TIPN from initiation of the stated line of therapy. ${ }^{\mathrm{c}}$ Includes lenalidomide/thalidomide-based regimens.

\section{Treatment Patterns and Occurrence of TIPN by Line of Treatment}

Overall (irrespective of TIPN status), the most common treatments in the first-line $(n=457)$ and second-line $(n=212)$ settings were bortezomib- $(69 \%)$ and lenalidomide- (49\%) based regimens, respectively; similar observations were made for patients with and without TIPN (Table 2). Bortezomib-based regimens were used in $40 \%$ of patients in the third/fourth lines $(n=87)$ of treatment, with similar proportions observed irrespective of TIPN status. Lenalidomide-based regimens were used in $48 \%$ of patients with TIPN and $30 \%$ of those without TIPN undergoing third/fourth-line treatment.
Of the 102 cases of TIPN, 57 (56\%) were reported in the first-line setting (Table 3). Most of the cases of TIPN reported during first-line treatment occurred in patients receiving bortezomib-based therapy (48/57 cases; $84 \%$ ). Overall, 48/314 (15\%) patients receiving bortezomibbased therapy in the first-line setting experienced TIPN (Tables 2, 3). During second-line treatment, 19/31 (61\%) of the reported cases of TIPN occurred in patients receiving immunomodulatory drug (IMiD)-based (lenalidomide or thalidomide) regimens (Table 3). Overall, 19/104 (18\%) patients receiving lenalidomide-based second-line treatment experienced TIPN (Tables 2, 3). Although fewer patients received bortezomib-based second-line 
Table 4. Characteristics of patients with TIPN and matched patients with no TIPN

\begin{tabular}{|c|c|c|c|}
\hline Variable & $\begin{array}{l}\text { Patients with TIPN } \\
(n=73)\end{array}$ & $\begin{array}{l}\text { Matched patients } \\
\text { with no TIPN }(n=73)\end{array}$ & $p$ value \\
\hline Sex, male, $n(\%)$ & $48(65.8)$ & $48(65.8)$ & $1.0000^{\mathrm{a}}$ \\
\hline \multicolumn{4}{|l|}{ ISS stage, $n(\%)$} \\
\hline I & $9(12.3)$ & $7(9.6)$ & \multirow[t]{3}{*}{$0.7130^{\mathrm{a}}$} \\
\hline II & $21(28.8)$ & $17(23.3)$ & \\
\hline Missing & $36(49.3)$ & $43(58.9)$ & \\
\hline \multicolumn{4}{|l|}{ Lab values, $[n]$ mean $(\mathrm{SD})$} \\
\hline Calcium, mg/dL & [44] $9.7(1.2)$ & [51] $9.6(1.1)$ & $0.5553^{\mathrm{b}}$ \\
\hline $\mathrm{Cr}, \mu \mathrm{mol} / \mathrm{L}$ & [48] $145.5(173.8)$ & [50] $126.9(134.1)$ & $0.7491^{\mathrm{b}}$ \\
\hline Hemoglobin, g/dL & [47] $11.9(1.8)$ & [51] $11.7(1.2)$ & $0.8337^{\mathrm{b}}$ \\
\hline B2-microglobulin, mg/L & [37] $5.1(5.4)$ & [30] $3.9(2.1)$ & $0.9246^{\mathrm{b}}$ \\
\hline \multicolumn{4}{|l|}{ Regimen inducing PN/matched treatment, $n(\%)$} \\
\hline Bortezomib + thalidomide based & $1(1.4)$ & $1(1.4)$ & \multirow{3}{*}{$1.0000^{\mathrm{a}}$} \\
\hline Lenalidomide based & $3(4.1)$ & $3(4.1)$ & \\
\hline Thalidomide based & $5(6.8)$ & $5(6.8)$ & \\
\hline \multicolumn{4}{|l|}{ Second line } \\
\hline Bortezomib based & $5(6.8)$ & $5(6.8)$ & \multirow[t]{2}{*}{$0.9742^{\mathrm{a}}$} \\
\hline Lenalidomide based & $12(16.4)$ & $11(15.1)$ & \\
\hline \multicolumn{4}{|l|}{ Third line } \\
\hline Bortezomib based & $1(1.4)$ & $2(2.7)$ & \multirow[t]{2}{*}{$0.8434^{\mathrm{a}}$} \\
\hline Lenalidomide based & $2(2.7)$ & $2(2.7)$ & \\
\hline Time from MM diagnosis to initiation of treatment inducing PN/ & & & \\
\hline matched treatment, median (range), months & $0.2(0.0-7.9)$ & $0.1(0.0-7.2)$ & $0.5676^{\mathrm{b}}$ \\
\hline Follow-up time from TIPN date/pseudo TIPN date, median (range), years & $1.7(0.1-7.1)$ & $1.8(-2.3$ to 8.1$)$ & $0.7188^{b}$ \\
\hline
\end{tabular}

ISS, International Staging System; MM, multiple myeloma; SCT, stem cell transplantation; TIPN, treatment-induced peripheral neuropathy; SD, standard deviation. ${ }^{a} \chi^{2}$ square test. ${ }^{b}$ Kruskal-Wallis test. ${ }^{c}$ Patients with any evidence of prior TIPN in the available patient records.

therapy, a similar proportion developed TIPN (12/65; $18 \%)$.

For bortezomib-based regimens, median time to TIPN from initiation of treatment decreased with increasing line of therapy (Table 3). No clear trend in time to TIPN was observed with IMiD-based therapy.

Of the 102 patients experiencing TIPN in this study, 49 (48\%) had a TIPN diagnosis before and 53 (52\%) patients had a TIPN diagnosis after the date of approval of subcutaneous bortezomib in Sweden. The proportion of patients with a prior TIPN diagnosis in their history was $9 \%$ after and $2 \%$ before the subcutaneous bortezomib approval date.

Burden of Treatment-Induced Peripheral Neuropathy in Multiple Myeloma

\section{TIPN-Associated HCRU and Costs}

A total of 73 patients with TIPN could be matched sufficiently well to patients without TIPN and so were included in the analyses comparing HCRU use between patients with and without TIPN. There were no statistically significant differences found between matched patients with and without TIPN, in terms of ISS stage, SCT status, and time to induction therapy (Table 4).

Overall, in the matched analysis, patients with TIPN had increased HCRU compared with those without TIPN (Table 5). Patients with TIPN had more hospital outpatient visits than those without TIPN (mean 17.8 vs. 12.6 visits, respectively; $p=0.0031$ ); the number of hospitalizations was not significantly different between groups 
Table 5. HCRU and costs for patients with TIPN and matched patients with no TIPN

\begin{tabular}{|c|c|c|c|c|}
\hline Variable & $\begin{array}{l}\text { Patients with } \\
\text { TIPN }(n=73)\end{array}$ & $\begin{array}{l}\text { Matched patients } \\
\text { with no TIPN }(n=73)\end{array}$ & $\begin{array}{l}\text { Mean difference of } \\
\text { matched pairs (SD) }\end{array}$ & $p$ value $^{\mathrm{a}}$ \\
\hline Hospitalizations per patient-year, mean (SD), $n$ & $3.5(8.0)$ & $2.3(4.9)$ & $1.2(9.1)$ & 0.1264 \\
\hline Cost per patient-year, mean (SD), EUR & $16,316.2(55,568.1)$ & $6,609.3(16,763.1)$ & $6,079.4(32,122.6)$ & 0.0805 \\
\hline Hospital outpatient visits per patient-year, mean (SD), $n$ & $17.8(16.8)$ & $12.6(19.8)$ & $5.2(25.3)$ & 0.0031 \\
\hline Cost per patient-year, mean (SD), EUR & $8,638.0(7,953.5)$ & $5,776.5(8,703.6)$ & $2,512.9(7,354.1)$ & 0.0014 \\
\hline
\end{tabular}

TIPN, treatment-induced peripheral neuropathy; HCRU, healthcare resource utilization; SD, standard deviation. ${ }^{a}$ Kruskal-Wallis test. ${ }^{b}$ Included the cost of all drugs dispensed from the pharmacy; any other drugs, for example, drugs given in hospital for the treatment of MM, were captured in the total costs.

(mean 3.5 vs. 2.3 visits; $p=0.1264$ ). There was a trend towards increased total length of hospitalization in patients with TIPN versus those without TIPN (mean 26.2 vs. 20.6 days; median 7.2 vs. 1.2 days; $p=0.0938$ ).

Patients with TIPN also incurred significantly increased total costs per patient-year than those without TIPN (mean EUR 52,353 vs. EUR 31,194, respectively; $p=0.0007$ ) (Table 5). This was mainly driven by the additional cost of outpatient visits (mean EUR 8,638 vs. EUR 5,777; $p=0.0014$ ) and prescription drugs (mean EUR 27,399 vs. EUR 18,809; $p=0.0022$ ) in the TIPN versus non-TIPN cohorts, respectively.

\section{Discussion}

This retrospective cohort study used EMR data from patients with MM from 3 large Swedish hematology clinics to assess the occurrence and economic burden associated with TIPN in clinical practice. Overall, TIPN was reported in $22 \%$ of patients, mostly in those receiving bortezomib- or lenalidomide-based regimens. In the matched analyses, patients with TIPN had increased HCRU and higher costs per patient-year, compared with those without TIPN.

The overall rate of TIPN reported here is slightly higher than that reported in a recent US publication (15.5\% [26]), but lower than another European real-world report (45\% [27]) and a US community-based study (53\% [28]). The rate of grade $\geq 3$ TIPN reported in the communitybased study was $20 \%$ - similar to the TIPN incidence reported here. It is possible that only the more severe TIPN cases that required treatment were captured in our study. Cases of grade 1/2 TIPN usually do not require interven- tion and so may not have been noted in the EMR. Information on TIPN grade/severity was not captured. These differences in rates may also relate to variations in patient selection, follow-up period, methodology used to define the occurrence of TIPN, and between-country differences in treatment practice. Nonetheless, taken together, these data suggest that TIPN remains a significant problem in patients with MM. In line with previous reports [8, 29], pre-existing TIPN is significantly associated with the development of grade $2 / 3$ TIPN [30]. In our study, $94 \%$ of the 102 patients experiencing TIPN had no prior history of TIPN in their EMR, which may also help explain the relatively low TIPN rates.

The economic impact of TIPN reported here is consistent with reports from the USA in patients with MM [26] and solid tumors [25], which indicated that patients with TIPN incur greater costs than those without TIPN. In the previous MM study, total costs per patient per month were significantly higher (by USD 1,509) for patients with TIPN than for controls [26]. In our study, the mean additional total cost associated with TIPN was EUR 17,183 per patient-year. This was mostly driven by increased outpatient visits and prescription drug costs; in previous studies, patients with TIPN also required significantly more hospitalizations. This may indicate a potential difference in TIPN management between the USA and Sweden. There were no clear differences in the type of drugs prescribed between patients with/without TIPN in our analyses (data not shown), but the number of patients receiving no prescriptions (therefore zero prescription costs) was higher in patients without TIPN and this contributed to the cost differences between groups. If our observed TIPN rates reflect only the more severe cases, the reported costs associated with TIPN may be higher than 
would be expected if all TIPN grades were represented. Conversely, severe TIPN may result in reduced dosing or discontinuation of costly anti-MM medication and therefore lower costs but also potentially reduced survival. Furthermore, costs were not adjusted for bortezomib usage. As this agent induces TIPN and requires frequent hospital visits, patients undergoing bortezomib treatment may be expected to encounter increased costs. In some patients with more severe conditions such as renal failure, 1 confounding factor could be more intensive treatment with bortezomib and therefore increased costs and hospitalizations. Unfortunately, in our study, the available data on disease severity are incomplete and therefore make it difficult to assess the impact of disease severity upon costs. Costs would also probably be impacted by the bortezomib formulation used because switching from intravenous to subcutaneous bortezomib would likely reduce the frequency of TIPN [19-21]. A limitation of our study is that the type of bortezomib formulation used was not formally captured. Nonetheless, it is likely that many patients receiving bortezomib would have switched to the subcutaneous formulation during the observation period (20062017). A post hoc sub-analysis was performed to determine the number of patients experiencing TIPN before and after the date when subcutaneous bortezomib became available for use in Sweden (September 28, 2012). Of the 102 patients experiencing TIPN in our study, similar proportions had a TIPN diagnosis before and after this cut-off date (48 vs. $52 \%$, respectively). However, this post hoc analysis could be confounded by the speed of uptake of subcutaneous bortezomib in Sweden and also by the proportion of patients with a prior TIPN diagnosis in their history, which was higher in patients diagnosed with TIPN after versus before the subcutaneous bortezomib approval date ( 9 vs. $2 \%$, respectively).

As we report country-specific data for Sweden here, it is not possible to extrapolate these results to other countries due to differences in costs of hospitalizations, technical interventions (e.g., nerve conduction assays) and medications. Nonetheless, a strength of our study is that it provides real-world data on the management and outcomes of a large population of patients with MM in clinical practice. Our results should be representative of those in Sweden as a whole as the population and time period covered largely overlap with those included in a Swedish Myeloma Registry study, which comprised $97 \%$ of all patients diagnosed with MM in Sweden over that period (2008-2015) [7]. Overall, the patients in our study were slightly younger (median ages 66/68 years in patients with/without TIPN, respectively, vs. 71 years) and more

Burden of Treatment-Induced Peripheral

Neuropathy in Multiple Myeloma received first-line bortezomib- or IMiD-based treatment ( 95 vs. $68 \%$ ) than those in the previous report. However, usage of these agents in patients aged $\leq 80$ years in the previous study $(73-81 \%$ [7]) was closer to the rates reported here. Furthermore, patients experiencing TIPN in our study were significantly younger $(p=0.0169)$ than those without TIPN, which may suggest more intensive regimens (e.g., SCT and melphalan-containing regimens or a twice-weekly bortezomib) are used in younger patients with MM.

An intrinsic limitation of EMR-based studies is that these records are not intended for study purposes and the data contained within them are restricted to that which was documented in clinical practice. Although validation work is ongoing by health authorities to ensure that data in national registries are complete and of high quality, in our study the diagnosis coding available was sometimes not as detailed as desired. In addition, as many EMRbased variables were derived from free-text searches, the completeness of data extracted depended on appropriate formulation of the searches and how well the information was documented in the system. To help ensure optimum information recovery, searches were performed in close collaboration with clinicians who were familiar with EMR data entry. Nonetheless, any missing data are expected to be random, and so should not bias the overall results of this study. Furthermore, data on diagnoses and procedures performed in primary care were not included, which may have led to an underestimation of HCRU and costs, as well as the prevalence of some comorbidities (e.g., diabetes). An additional limitation is that bortezomib was only indicated for use in exceptional circumstances in Europe until 2012 and so the proportion of patients treated with this agent for the first part of this study may be lower than would be observed today.

In summary, these real-world data from Sweden suggest that bortezomib- and IMiD-based treatment is often associated with TIPN in patients with MM. Our results also indicate that patients with TIPN require more frequent outpatient visits, resulting in greater costs, compared with patients without TIPN. In the future, the use of effective treatments for MM that are associated with a reduced incidence of TIPN could result in decreased healthcare expenditure.

\section{Acknowledgements}

Medical writing assistance was provided by Dawn Batty $\mathrm{PhD}$ (Bioscript Medical Ltd., Macclesfield, UK) and funded by Amgen (Europe) GmbH (Rotkreuz, Switzerland). 


\section{Statement of Ethics}

Ethics approval for this study was obtained from the Swedish Ethics Board in Stockholm (approval number: 2016/751-31/1; approval number for study amendment: 2017/544-32) and the need for written and informed consent was waived by the Swedish Ethics Board (Stockholm, Sweden).

\section{Conflict of Interest Statement}

H.N., M.H., and G.W. have no conflicts of interest to declare. Y.Q. and A.L. are employees of IQVIA; IQVIA received research grants from Amgen to conduct this research. V.P., I.M., L.K., E.H.F., and M.K.S. are Amgen employees and stockholders.

\section{Funding Sources}

This study was funded by Amgen (Europe) GmbH (Rotkreuz, Switzerland).

\section{Author Contributions}

H.N., G.W., and M.H. contributed to the interpretation of study data. A.L. contributed to the study design and the analysis and interpretation of study data. V.P., L.K., and Y.Q. were involved in the analysis and interpretation of study data. I.M., E.H.F., and M.K.S. contributed to the study design and interpretation of the data. All authors were involved in drafting the paper and/or revising it critically for important intellectual content, approved the final draft, and agreed to be accountable for all aspects of the work.

\section{Data Sharing Statement}

The data that have been used in this study are confidential registry data. Access to the study data is restricted to the study team and is only to be used as stated in the ethics application. The study data will be destroyed after project completion.

\section{References}

1 Palumbo A, Anderson K. Multiple myeloma. N Engl J Med. 2011 Mar;364(11):104660.

2 Ferlay J, Soerjomataram I, Dikshit R, Eser S, Mathers C, Rebelo M, et al. Cancer incidence and mortality worldwide: sources, methods and major patterns in GLOBOCAN 2012. Int J Cancer. 2015 Mar;136(5):E359-86.

3 NORDCAN AotNCR. Cancer stat factsheets: Sweden: multiple myeloma. 2019.

4 Smith A, Wisloff F, Samson D. Guidelines on the diagnosis and management of multiple myeloma 2005. Br J Haematol. 2006 Feb; 132(4):410-51.

5 Palumbo A, Rajkumar SV, San Miguel JF, Larocca A, Niesvizky R, Morgan G, et al. International Myeloma Working Group consensus statement for the management, treatment, and supportive care of patients with myeloma not eligible for standard autologous stem-cell transplantation. J Clin Oncol. 2014 Feb;32(6):587-600.

6 Moreau P, San Miguel J, Sonneveld P, Mateos MV, Zamagni E, Avet-Loiseau H, et al. Multiple myeloma: ESMO Clinical Practice Guidelines for diagnosis, treatment and follow-up. Ann Oncol. 2017 Jul;28(Suppl 4): iv52-61.

7 Blimark CH, Turesson I, Genell A, Ahlberg L, Björkstrand B, Carlson K, et al. Outcome and survival of myeloma patients diagnosed 2008-2015. Real-world data on 4904 patients from the Swedish myeloma registry. Haematologica. 2018 Mar;103(3):506-13.

8 Mohty B, El-Cheikh J, Yakoub-Agha I, Moreau P, Harousseau JL, Mohty M. Peripheral neuropathy and new treatments for multiple myeloma: background and practical recommendations. Haematologica. 2010 Feb; 95(2):311-9.
9 Richardson PG, Delforge M, Beksac M, Wen $\mathrm{P}$, Jongen JL, Sezer O, et al. Management of treatment-emergent peripheral neuropathy in multiple myeloma. Leukemia. 2012 Apr; 26(4):595-608.

10 Watson JC, Dyck PJ. Peripheral neuropathy: a practical approach to diagnosis and symptom management. Mayo Clin Proc. 2015 Jul; 90(7):940-51.

11 Richardson PG, Laubach JP, Schlossman RL, Mitsiades C, Anderson K. Complications of multiple myeloma therapy, part 1: risk reduction and management of peripheral neuropathy and asthenia. J Natl Compr Canc Netw. 2010 Feb;8(Suppl 1):S4-12.

12 Beijers AJ, Vreugdenhil G, Oerlemans S, Eurelings M, Minnema MC, Eeltink CM, et al. Chemotherapy-induced neuropathy in multiple myeloma: influence on quality of life and development of a questionnaire to compose common toxicity criteria grading for use in daily clinical practice. Support Care Cancer. 2016 Jun;24(6):2411-20.

13 Richardson PG, Briemberg H, Jagannath S, Wen PY, Barlogie B, Berenson J, et al. Frequency, characteristics, and reversibility of peripheral neuropathy during treatment of advanced multiple myeloma with bortezomib. J Clin Oncol. 2006 Jul;24(19):311320.

14 Walker MS, Cong Z, Khopf KB, Aggarwal SKKJ, Houts AC. Real world impact of treatment-induced perpheral neuropathy (TIPN) on patient reported outcomes (PROs) in patients with multiple myeloma (MM) in the United States. J Clin Oncol. 2015;33(Suppl 15):e19531.

15 Meyer-Rosberg K, Kvarnström A, Kinnman E, Gordh T, Nordfors LO, Kristofferson A. Peripheral neuropathic pain: a multidimen- sional burden for patients. Eur J Pain. 2001; 5(4):379-89.

16 Delforge M, Bladé J, Dimopoulos MA, Facon T, Kropff M, Ludwig H, et al. Treatment-related peripheral neuropathy in multiple myeloma: the challenge continues. Lancet Oncol. 2010 Nov;11(11):1086-95.

17 Jagannath S, Barlogie B, Berenson J, Siegel D, Irwin D, Richardson PG, et al. A phase 2 study of two doses of bortezomib in relapsed or refractory myeloma. Br J Haematol. 2004 Oct; 127(2):165-72.

18 Richardson PG, Sonneveld P, Schuster MW, Irwin D, Stadtmauer EA, Facon T, et al. Bortezomib or high-dose dexamethasone for relapsed multiple myeloma. N Engl J Med. 2005 Jun;352(24):2487-98.

19 Moreau P, Pylypenko H, Grosicki S, Karamanesht I, Leleu X, Grishunina M, et al. Subcutaneous versus intravenous administration of bortezomib in patients with relapsed multiple myeloma: a randomised, phase 3, noninferiority study. Lancet Oncol. 2011 May; 12(5):431-40.

$20 \mathrm{Hu}$ B, Zhou Q, Wu T, Zhuang L, Yi L, Cao J, et al. Efficacy and safety of subcutaneous versus intravenous bortezomib in multiple myeloma: a meta-analysis. Int J Clin Pharmacol Ther. 2017 Apr;55(4):329-38.

$21 \mathrm{Mu}$ SD, Ai LS, Qin Y, Hu Y. Subcutaneous versus intravenous bortezomib administration for multiple myeloma patients: a metaanalysis. Curr Med Sci. 2018 Feb;38(1):43-50.

22 Moreau P, Pylypenko H, Grosicki S, Karamanesht I, Leleu X, Rekhtman G, et al. Subcutaneous versus intravenous bortezomib in patients with relapsed multiple myeloma: subanalysis of patients with renal impairment in the phase III MMY-3021 study. Haematologica. 2015 May; 100(5):e207-10. 
23 Jakubowiak A. Novel therapies for relapsed/ refractory multiple myeloma: how can we improve on "salvage" therapy?: introduction. Semin Hematol. 2012 Jul;49(Suppl 1):S1-2.

24 Martin TG III, Panjabi S, Kerr J, Martin MG, Walker MS. Association of treatment induced peripheral neuropathy (TIPN) with treatment patterns and outcomes in patients (pts) with newly diagnosed multiple myeloma (NDMM). Blood. 2013 Sep;122(21):1750-60.

25 Pike CT, Birnbaum HG, Muehlenbein CE, Pohl GM, Natale RB. Healthcare costs and workloss burden of patients with chemotherapy-associated peripheral neuropathy in breast, ovarian, head and neck, and nonsmall cell lung cancer. Chemother Res Pract. 2012 Mar;2012:913848.
26 Song X, Wilson KL, Kagan J, Panjabi S. Cost of peripheral neuropathy in patients receiving treatment for multiple myeloma: a US administrative claims analysis. Ther Adv Hematol. 2019 Apr;10:2040620719839025.

27 Yong K, Delforge M, Driessen C, Fink L, Flinois A, Gonzalez-McQuire S, et al. Multiple myeloma: patient outcomes in real-world practice. Br J Haematol. 2016 Oct;175(2): 252-64.

28 Niesvizky R, Flinn IW, Rifkin R, Gabrail N, Charu V, Clowney B, et al. Community-based phase IIIB trial of three UPFRONT bortezomib-based myeloma regimens. J Clin Oncol. 2015 Nov;33(33):3921-9.
29 Garcia-Sanz R, Corchete LA, Alcoceba M, Chillon MC, Jiménez C, Prieto I, et al. Prediction of peripheral neuropathy in multiple myeloma patients receiving bortezomib and thalidomide: a genetic study based on a single nucleotide polymorphism array. Hematol Oncol. 2017 Dec;35(4):746-51.

30 Dimopoulos MA, Mateos MV, Richardson PG, Schlag R, Khuageva NK, Shpilberg O, et al. Risk factors for, and reversibility of, peripheral neuropathy associated with bortezomib-melphalan-prednisone in newly diagnosed patients with multiple myeloma: subanalysis of the phase 3 VISTA study. Eur J Haematol. 2011 Jan;86(1):23-31. 